\title{
Participación ciudadana y estrategias de comuni- cación en el gobierno central de El Salvador
}

Camila Calles Minero

Docente investigadora, Dirección de Investigaciones de la UTEC

\section{Resumen}

¿Qué estrategias de difusión utiliza el gobierno central para promover la participación ciudadana y qué incidencia tiene esta en el acontecer gubernamental? Esta investigación identificó esas estrategias y su inocencia, así como la incidencia de la participación ciudadana en la toma de decisiones del gobierno central de El Salvador. A través de entrevistas en profundidad, se logró establecer que en el gobierno central la participación ciudadana está enfocada en la dimensión de la denuncia; hacen falta mecanismos en los que realmente la población incida en la toma de decisiones gubernamentales. Además, los contados mecanismos de participación que existen no son divulgados adecuadamente, las instancias del gobierno no tienen estrategias de comunicación exclusivas para promoverlos.

\section{Palabras claves:}

Participación ciudadana, mecanismo de participación, medios de comunicación, estrategias de comunicación.

\begin{abstract}
What dissemination strategies does the central government use to promote citizen participation and what effect has it in government befall? This research identified these strategies and their innocence, as well as the impact of citizen participation in decision-making of central government of El Salvador. Through in-depth interviews, it was possible to establish that in the central government, citizen participation is focused only on the dimension of the complaint, are needed mechanisms in whose people actually affects in government decision making. Moreover, the few existing mechanisms for participation, are not disclosed properly, the government agencies do not have exclusive communication strategies to promote them.
\end{abstract}

\section{Key words:}

Citizen participation, participation mechanism, media, communication strategies.

\section{Introducción}

Idiccionario de la Real Academia
de la Lengua (2001) define que algo, esa acción se puede llevar hasta el interior de la dinámica social, y surge la participación ciudadana, pero hablar de las acciones dentro de la sociedad es más complejo que el hecho de tomar parte de algo. La participación ciudadana tiene como objetivo incidir de alguna manera, en el aparato del Estado, abonar en la construcción de políticas públicas. Para ello, el Estado tiene que entablar un diálogo con la ciudadanía. 


\section{ent erno}

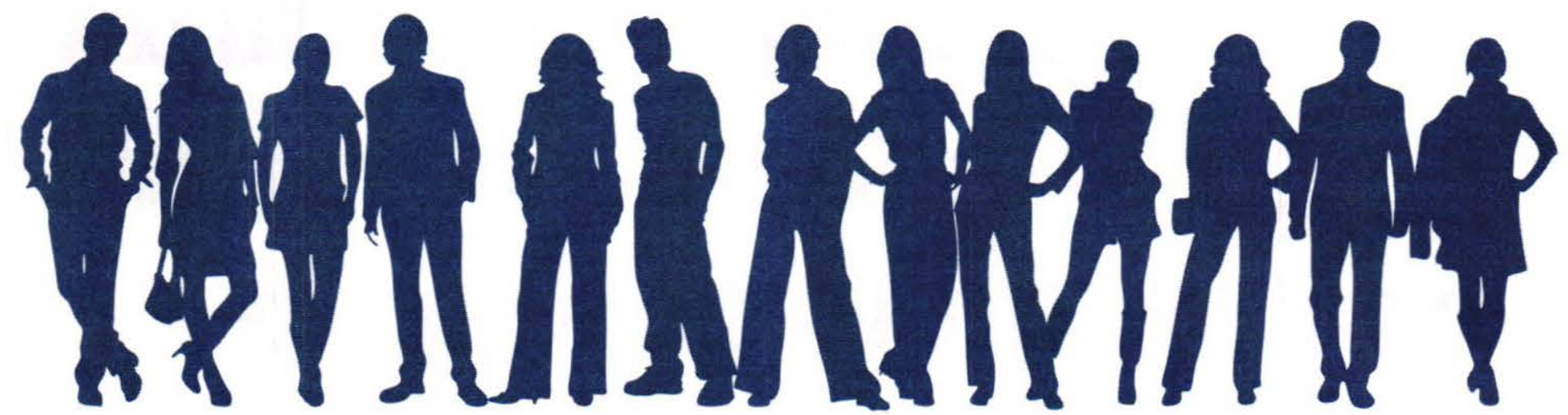

En El Salvador, la búsqueda de intervención por parte de la sociedad en la toma de decisiones se puede vislumbrar desde la tercera década del siglo pasado, cuando movimientos indígenas-campesinos intentaban cambiar la dinámica social. Otro ejemplo de participación fue la capacidad organizativa social, durante la década de los 80 . Sin embargo, estas manifestaciones eran de índole política y orientada a cambiar toda una estructura. Después de los Acuerdos de Paz (1992), la forma de incidir cambió, surgieron organizaciones civiles en áreas específicas, que se iniciaron en la construcción de discursos, en aras de cambiar las condiciones de vida en torno a temas concretos.

Azpuru, Blanco, Córdova, Loya, Ramos y Zapata (2007) establecen que, en el caso de El Salvador, la historia de la concertación es corta y las iniciativas que han logrado desarrollarse han sido frágiles, cambiantes y limitadas. El primer espacio de concertación que vivió - según esos autores- fue el Pacto de San Andrés, firmado en mayo de 1995 por el gobierno del presidente Armando Calderón Sol y el recién creado Partido Demócrata (PD), en donde se establecieron nuevas correlaciones de fuerza para la vida política en el país. Un año después de su firma, el PD anunció la ruptura del pacto debido al incumplimiento del gobierno.

La participación ciudadana es un proceso sistemático por medio del cual la ciudadanía se organiza para incidir en la vida pública nacional y municipal, a través de mecanismos e instrumentos que les permiten ser parte activa de la toma de decisiones, del control social y de la corresponsabilidad, actuando de manera asociada con sus gobernantes en aras de procurar el bien común y de construir una nueva cultura política democrática, de acuerdo con la Iniciativa Social para la Democracia (ISD, 2002).

El país cuenta con mecanismos en los que la población puede avocarse para incidir, de alguna manera, en la creación de políticas públicas, pero no todos los conocen ante la falta de una política de comunicación efectiva, para darlos a conocer y explicarlos, según Rivas (2009).

Para especialistas como Uceda (2005), actualmente existe un divorcio entre la actividad social, los gobiernos y las comunicaciones, sobre todo en potenciar los mecanismos de participación ciudadana ya estableci- dos, así como los caminos que buscan abrir las organizaciones sociales. En ese contexto, aparece el Internet como una herramienta que se puede utilizar para hacer efectivos los mecanismos de participación ciudadana, así como ampliar el alcance de las oficinas estatales, cuyas puertas están lejos de algunos ciudadanos. Las TIC suponen un punto de partida hacia facilitar la participación de los ciudadanos en la toma de decisiones públicas.

\section{Método}

La investigación se realizó en la 77 instancias gubernamentales, en las que se solicitó información sobre la existencia de mecanismos de participación ciudadana y oficinas de comunicación. Se entrevistó, en profundidad, a los encargados de las oficinas relacionadas con la participación ciudadana y del área de comunicaciones. Se realizó observación no estructurada de algunos procesos de participación. La mayor dificultad fue el acceso a la información, pues muchas instancias gubernamentales no dieron información pese a las múltiples solicitudes que se hicieron por diferentes vías. 


\section{Resultados}

En El Salvador existen mecanismos de participación ciudadana establecidos legalmente, en la Constitución de la República, en la Ley de Medio Ambiente, en el Código Municipal y la Ley de Creación del Fondo para el Desarrollo Económico y Social de los Municipios. Además, el país es firmante de la Convención Interamericana contra la Corrupción.

Esos apartados legales obligan a EI Salvador a crear espacios en donde los ciudadanos participen para incidir, de alguna manera u otra, en la toma de decisiones y creación de políticas públicas.

Los mecanismos más claramente identificados se encuentran en la Asamblea Legislativa, el Ministerio de Medio Ambiente y Recursos Naturales, el Tribunal Supremo Electoral. Otras instancias realizan, dentro de su propio trabajo, la apertura a la ciudadanía, como el caso del Instituto Salvadoreño para el Desarrollo de la Mujer (ISDEMU), la Defensoría del Consumidor, el Tribunal de Ética Gubernamental.

Sin embargo, no en todos la participación ciudadana significa que la población incide en la toma de decisiones del Estado, más bien significa un contacto con la ciudadanía. Este acercamiento es en temas de brindar información sobre la institución o para ser orientados en trámites específicos de la instancia gubernamental.

Aunque no es un mecanismo de participación directa, la creación de buzones de sugerencia en las entidades del Estado podría ser una forma de oír a la gente; solo el $33 \%$ de las instancias gubernamentales lo tienen de manera electrónica y el $1 \%$ de manera física en sus respectivas oficinas.

También están los mecanismos de participación que la misma ciudadanía crea: para el caso, las marchas, protestas, foros de discusión pública, cartas enviadas a funcionarios son parte de esas formas de participar.

Según la lista de instancias gubernamentales del Tribunal de Ética Gubernamental, existen 77 oficinas del Estado; de estas, el $55 \%$ establecieron claramente que no tenían mecanismos de participación de ningún tipo, ni buzones de sugerencias en sus oficinas, ni consultas a través de sus páginas electrónicas.

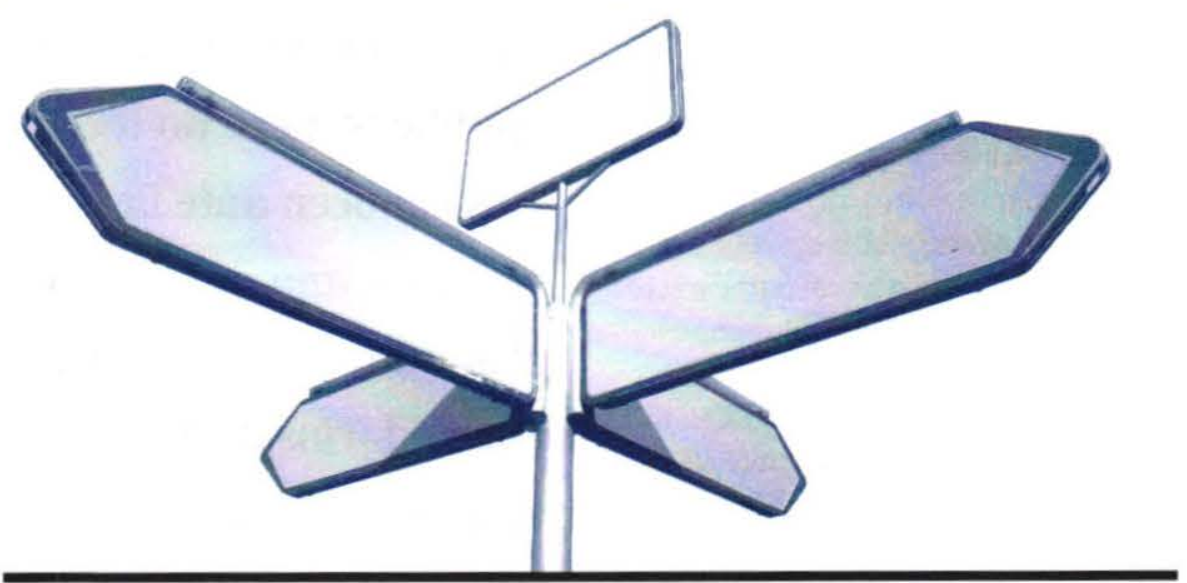

ce La participación ciudadana es un proceso sistemático por medio del cual la ciudadanía se organiza para incidir en la vida pública nacional y municipal, a través de mecanismos e instrumentos que les permiten ser parte activa de la toma de gy

El $37 \%$ de las instancias gubernamentales no respondieron la solicitud de información que se hizo de forma presencial, telefónica, cartas oficiales y correos electrónicos. Solo el $8 \%$ respondieron afirmativamente la existencia de mecanismos de participación ciudadana. Estas instancias gubernamentales fueron: Asamblea Legislativa, Ministerio de Medio Ambiente y Recursos Naturales, Tribunal Supremo Electoral, Tribunal de Ética Gubernamental, Defensoría del Consumidor, Instituto Salvadoreño para el Desarrollo de la Mujer y el Ministerio de Economía.

Durante la investigación, se detectó que las instituciones gubernamentales no tienen una estrategia de comunicación exclusiva para promover la participación ciudadana. Este tema es parte del trabajo de comunicación en general que tienen las institucio- 
nes. El tema de participación ciudadana es difundido desde el área de este tema dentro de las instituciones y es implementado por los mismos empleados de esa área no por comunicadores.

Igualmente, ninguna tiene evaluación del trabajo de comunicaciones en torno al tema de participación ciudadana; además, los portales electrónicos de las instituciones aún no son explotados para incentivar la participación ciudadana.

Con estos resultados, el gobierno salvadoreño se ubica en el escenario que plantea el Programa de las Naciones Unidad para el DesarroIlo (1998), y en donde asegura que América Latina y El Caribe han vivido épocas aquejadas por los males del despotismo y el populismo; esto generó una cultura que no solamente no incentiva la participación, sino que pretenden hacerla superflua.

En el gobierno central, existe la secretaria de comunicaciones de la presidencia, desde donde se dictan las formas de trabajo de las comunicaciones de las instancias gubernamentales, además, existen oficinas de comunicaciones destinadas a guiar la comunicación interna y externa de cada una de las instancias gubernamentales. Esa secretaría de comunicaciones no brindó información para esta investigación, por lo que no se pudo conocer qué lugar tiene la participación ciudadana en las directrices generales de las estrategias de comunicación, desde la máxima entidad.

De las instancias gubernamentales que brindaron información, solo una tenía una estrategia de comunicación que surgió de un estudio previo; las

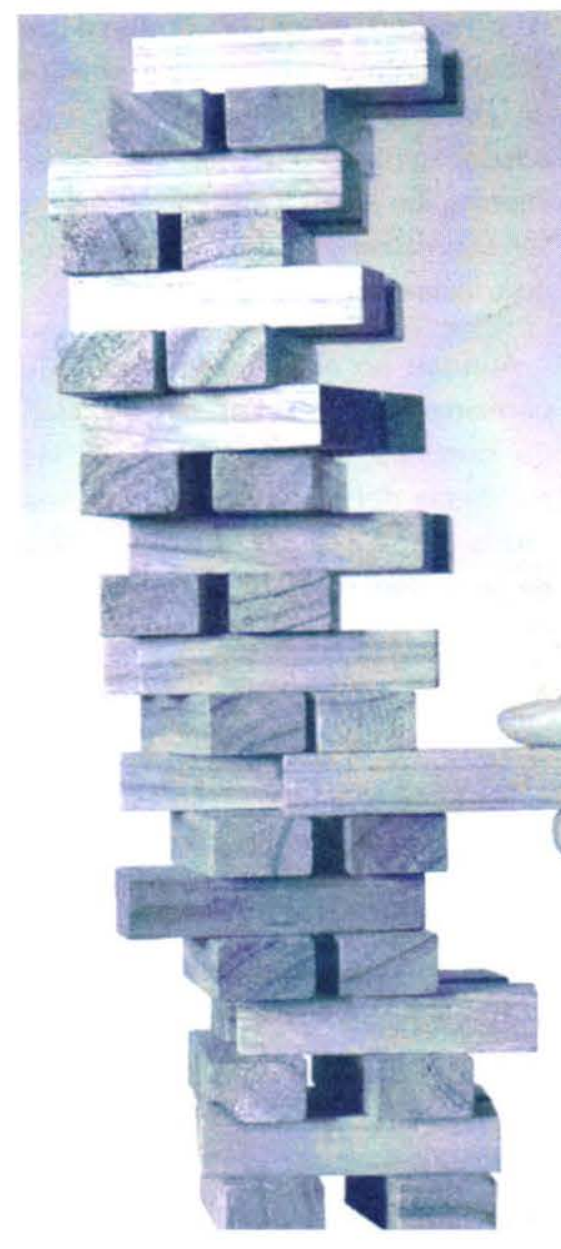

otras se basan en la necesidad de comunicar información institucional de las oficinas de gobierno. Además, solo una de las instancias del Estado tiene un diagnóstico de su trabajo en comunicaciones; las otras no tienen forma de evaluar cómo trabajan y el impacto que tiene su trabajo en el área de la participación ciudadana.

Ante la ausencia de las estrategias de comunicación, se refuerza la postura de Parrado y Löffler (2006), quienes establecen que la participación ciudadana es utilizada, al menos verbalmente, por políticos, funcionarios y organizaciones sociales. "Las fiestas locales organizadas por las asociaciones, barrios y el propio municipio proporcionan una buena ocasión

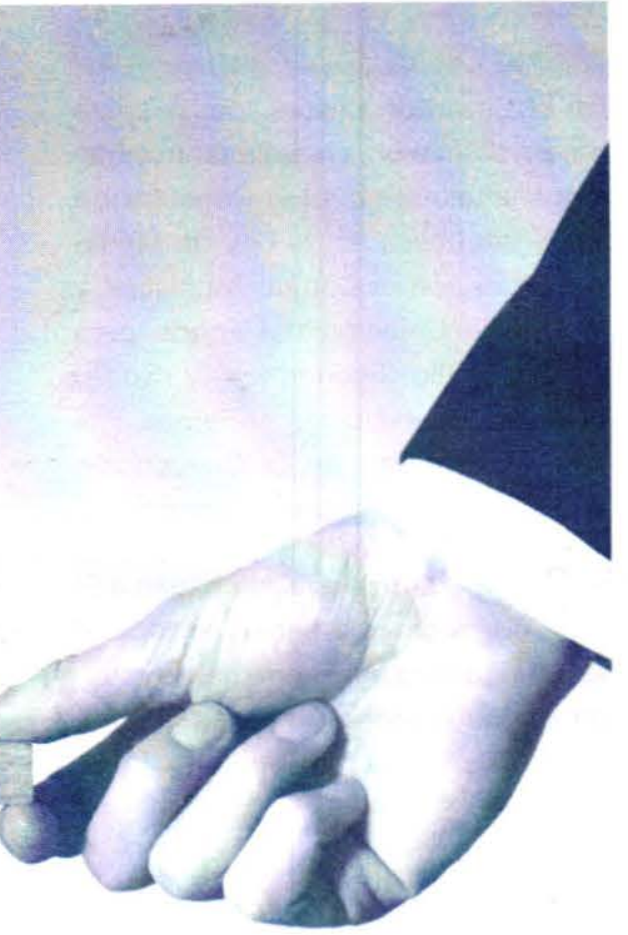

46 El país cuenta con mecanismos en los que la población puede avocarse para incidir, de alguna manera, en la creación de políticas públicas, pero no todos los conocen ante la falta de una política de comunicación efectiva, para darlos a conocer $y$ explicarlos. 
para salir en la foto, "cerca de los ciudadanos". Desgraciadamente, para muchos representantes políticos, la participación ciudadana finaliza en ese momento.

Las comunicaciones de las instancias gubernamentales tienen líneas amplias de trabajo, dadas por la tendencia de solo divulgar lo que hacen los titulares. Temas como la participación ciudadana son difundidos directamente por las áreas que las trabajan y, en algunas, con apoyo técnico de las oficinas de comunicaciones. La divulgación de los trabajos en participación ciudadana la realizan los mismos hacedores de esos trabajos y to las oficinas de comunicaciones.

Aunque las instancias gubernamentales tengan en sus páginas electrónicas formas en que los ciudadanos puedan consultar y solicitar información, estas no responden, al menos para efectos de esta investigación. Solo el $2 \%$ de las instituciones respondieron ante la petición de información desde sus portales virtuales.

Se detectaron problemas en la gestión de las comunicaciones. Por ejemplo, el Tribunal Supremo Electoral. La unidad de comunicaciones no se encarga ni de la publicidad, ni de la alimentación de la página electrónica. Ellos son los que tiene que asumir, desde una perspectiva comunicacional, el manejo de la imagen y el trabajo de la institución.

Al no tener estrategias de comunicación destinadas a la promoción y divulgación de la participación ciudadana, la población desconoce de los espacios que se tienen por ley y por iniciativa de las instancias, de proponer, participar e incidir. Es ne- cesario que exista comunicación de esos mecanismos, que la población se entere de cómo participar.

Los portales electrónicos son una forma viable de acercarse a la gente, pese a la brecha digital que existe. Se pueden crear una especia de oficinas virtuales en las cabeceras departamentales, para que desde ahí la gente tenga acceso a Internet y poder consultar, solicitar y proponer ante las instancias gubernamentales.

En ese marco, los mecanismos de participación ciudadana con los que cuentan la población son escasos en el gobierno central; se limitan a la Asamblea Legislativa y la Corte Suprema de Justicia, a las consultas que debe de realizar el Ministerio de Medio Ambiente y Recursos Naturales.

Sin embargo, muchas de las oficinas de gobierno tienen formas de hacer contacto con la población y no necesariamente son formas de participación ciudadana, pues la población no tiene la posibilidad de incidir políticamente en la toma de decisiones.
Esas puertas para ver y escuchar a la gente están centradas en la denuncia.

Debido a la falta de información, fue imposible evaluar la incidencia de la participación ciudadana en la toma de decisiones del gobierno central de El Salvador, pues solo la Asamblea Legislativa brindó datos; la Corte Suprema de Justicia no dio información y el MARN no tiene contabilizado el número de casos resueltos ante las denuncias, por la falta de una sistematización de información por parte de la administración anterior.

En el caso de la Asamblea Legislativa, durante el 2008 y el primer trimestre del 2009 , en el $50 \%$ de las leyes aprobadas hubo presencia de la población, pero hay que destacar que solo se aprobaron dos leyes, en la iniciativa de ley de una de ellas estaba la petición desde la población civil.

Ante esa carencia, una buena alternativa para el gobierno central salvadoreño es la mejor utilización del ciberespacio. Muchos autores lo catalogan como uno de los mejores

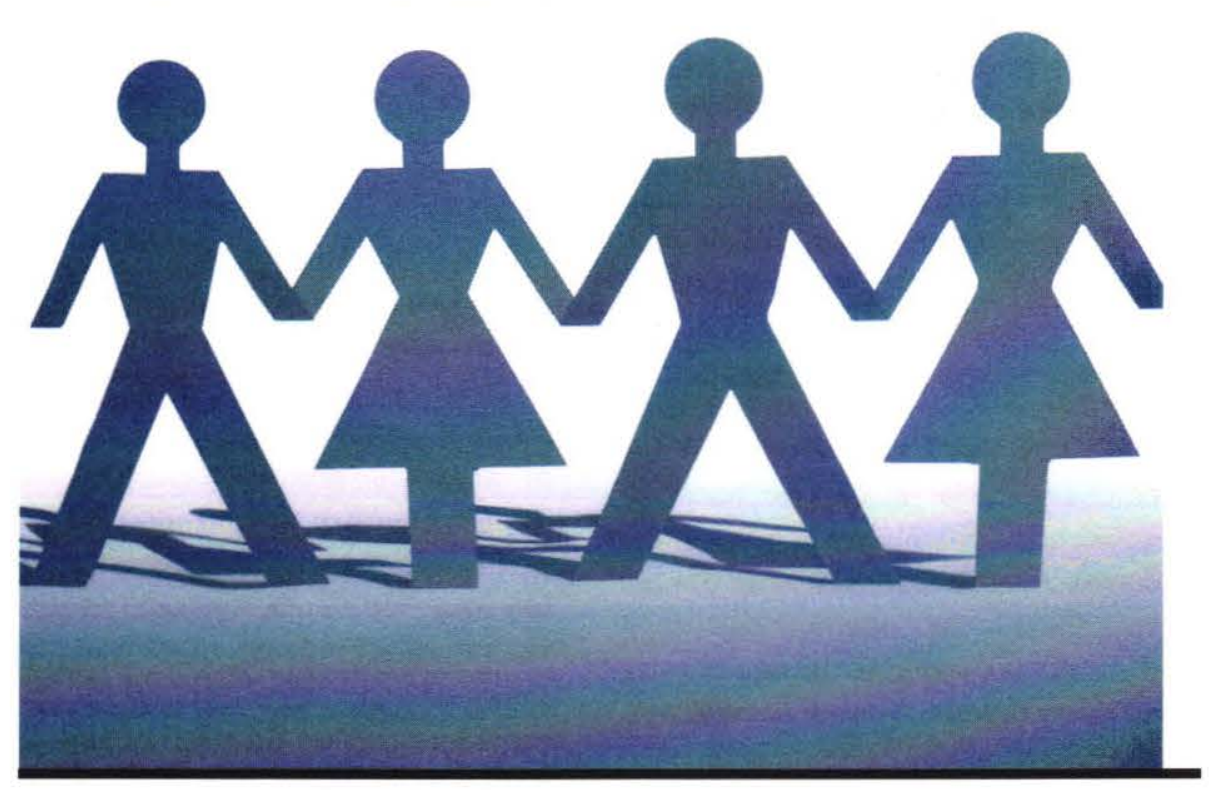




\section{ent orno}

(6)

Debido a la falta de información, fue imposible evaluar

la incidencia de la

participación ciudadana

en la toma de decisiones

del gobierno central de

EI Salvador, pues solo

la Asamblea Legislativa

brindó datos; la Corte

Suprema de Justicia

no dio información

y el MARN no tiene

contabilizado el número

de casos resueltos

ante las denuncias

por la falta de una

sistematización de información.y

areópagos políticos que permite la comunicación, pero no solo el hecho de colocar la Internet en medio de los gobernantes y los ciudadanos provocará la participación ciudadana; más bien el espíritu democrático con el que se conciban las herramientas, así darán apertura a la ciudadanía, tal como lo establece López (2001).

El gobierno salvadoreño debe entonces iniciar el camino hacia el gobierno electrónico, generando con ello otros mecanismos en donde los ciudadanos puedan participar e incidir en la toma de decisiones, así como poder proponer mejores políticas públicas.

\section{Propuesta}

Ante los hallazgos de esta investigación, se propone la creación de una política nacional de participación ciudadana, en donde se incluya una estrategia de comunicacióngubernamental para dar a conocer los mecanismos con los que cuentan los salvadoreños, para incidir políticamente; pero, además, crear más mecanismos de participación ciudadana.

La estrategia de comunicación estará guiada por esta política y tendrá que hacer énfasis en la divulgación de las formas que existen de participar $y$ la forma en que deben de ser utilizados para incidir en la toma de decisiones del Estado. Además, periódicamente se tendrá que dar a conocer los resultados de la participación de las personas; es decir, mostrar las cifras de cuántos han participado y qué se ha logrado con esa participación. Esta difusión podría ser en las mismas instancias del gobierno, a través de hojas volantes, trípticos o afiches colocados en las oficinas para que los usuarios puedan verlos.

Esta política nacional de participación ciudadana debe de tener, además, un componente relativo al fomento de una cultura de participación para ello y desde el área de comunicaciones; es decir, siempre en la estrategia comunicacional, fomentar los valores y la necesidad de involucrarse, para crear en la población la costumbre de utilizar los mecanismos de participación. Para ello se tiene que crear esos mecanismos en dondela población pueda acercarse y proponer, en las instancias gubernamentales.

A través de la política nacional de participación ciudadana, el gobierno central puede impulsar el gobierno electrónico, fortaleciendo sus páginas electrónicas para que estas permitan una real participación de la ciudadanía, en el sentido de que, a través de estos portales electrónicos, puedan enviar sus aportaciones para un mejor trabajo gubernamental, o simplemente, puedan consultar sobre el quehacer institucional.

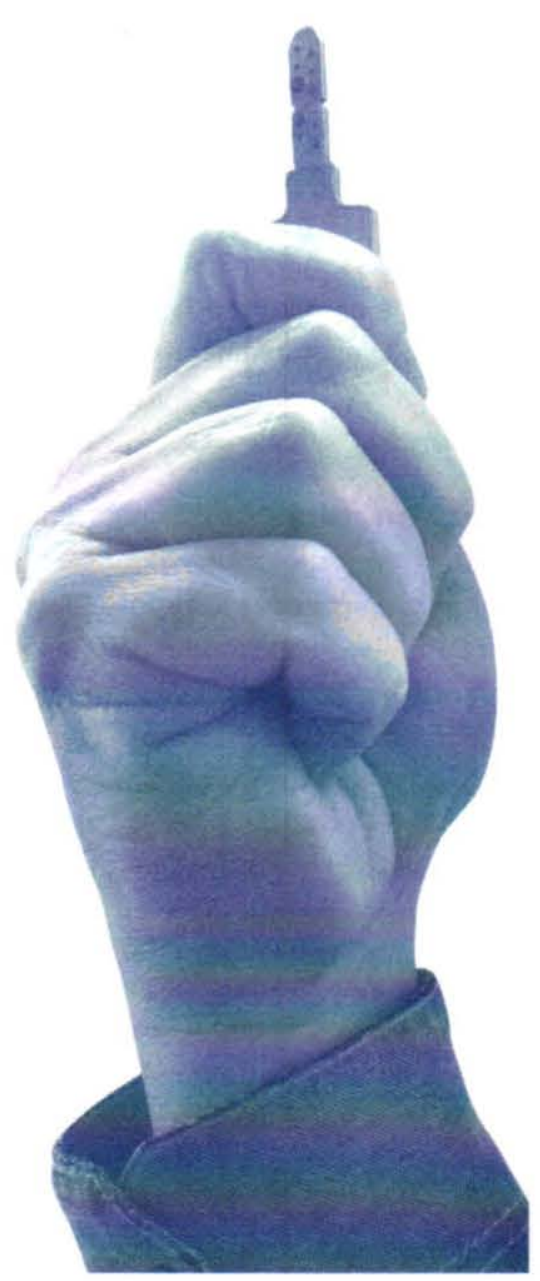


Pero, además de activar estos mecanismos, periódicamente se tiene que mostrar la incidencia de la población en la toma de decisiones. Se tiene que hacer publicidad de la efectividad de la participación ciudadana. La imagen de las instituciones gubernamentales tiene que ser manejada desde las unidades de comunicaciones.

Se propone también la confección de una guía de participación ciudadana, en la que se contemplen todos los mecanismos de participación ciudadana y que sea distribuida

\section{Bibliografía}

- Azpuru, D., Blanco, L., Córdova R., Loya N., Ramos C., \& Zapata A., (2007). Construyendo la democracia en sociedades postconflicto, un enfoque comparado entre Guatemala y EI Salvador, F\&G Editores.

- Colombo, C., (2005). Participación ciudadana en la red, recuperado en abril, 22, 2009 de http://www. gestioncultural.org/gc/boletin/pdf/ bgc11-CColombo.pdf.

- Cubías, A. (2009), subdirectora de Iniciativa Social para la Democracia -ISD-, comunicación personal, 28 de mayo 2009.

- Diccionario Real Academia de la Lengua (2001). Vigésima segunda edición.

- Góchez, R. E. (2008). Opinando públicamente. Talleres Imprenta Criterio 2008. San Salvador, El Salvador. en las oficinas estatales a todos los usuarios.

También se puede hacer algo desde la Universidad Tecnológica de El Salvador y con ello cumplir su misión de promover los cambios fundamentales que la sociedad necesita y continúe con la visión de ser reconocida por su capacidad de innovación. Podría contribuir con el fomento de la participación ciudadana en EI Salvador y en la creación de una cultura de participación. Esta universidad puede hacer un trabajo relevante: generación

- ISD (2002).Participación ciudadana y cultura política democrática. $1^{\mathrm{a}}$. Edición.

- Izurieta, R., Perina, R., \& Arterton, C. (2001) Estrategias de comunicación para gobierno. Editorial Ecuador. Quito, Ecuador.

- López, E. (2001). Comunicación, participación ciudadana y nuevas tecnologías. Recuperadoelabril,21, 2009 de http://dialnet.unirioja.es/ servlet/articulo codigo $=206590$.

- Parrado S. y Löffler E., (2006). Buenas y malas prácticas de participación ciudadana en Europa. recuperado en abril 21, 2009, de http://vlex.com/vid/buenasmalas-prácticas-participaciónciudadana-321441.

- PNUD, (1998). Evaluación del programa de gobernabilidad para América Latina y El Caribe. (Cáp. IV). de propuestas concretas y con acciones educativas destinadas a ello.

Desde la academia, se puede crear un equipo multidisciplinario entre las escuela de derecho y comunicaciones, para proponer la apertura de nuevos mecanismos de participación. Igualmente en la universidad se puede institucionalizar una semana de la participación ciudadana, en donde sé de información a la población y se analice el tema de la participación ciudadana.

- Rivas, Y. (2009). Directora de comunicaciones de Iniciativa Social para la Democracia -ISD, comunicación personal, 28 de mayo del 2009.

- Uceda. R, Orbe Y. Memoria de ponencias seminario taller iberoamericano. "Comunicación, participación ciudadana y medios de comunicación social (2005). Fundación Ayuda en Acción. Asociación de Periodistas de EI Salvador.

- Vall, F. P., \& Hurbe, M. (2005). Parlamento y comunicación (nuevos retos). Editorial Tecnos. Grupo Anaya. Madrid, España.

- Vivas T. D. (1997). La Participación ciudadana un proceso en construcción. Colombia. Imprenta nacional de Colombia. 